\section{Nutritionat of Metabolism}

\title{
My Meeting Report
}

I organized the $8^{\text {th }}$ Scientific Meeting of the Japanese Society of Anti-Aging Medicine with the title, 'The science for Anti-Aging Medicine: From Basic Research to Clinical Medicine', at the Tokyo International Forum, Jun 06-07, 2008.

The membership of the society has been increasing year by year and has surpassed 5,500 members as of 2008. Over 2,400 participants attended the $8^{\text {th }}$ Scientific Meeting, which was very active compared with other medical meetings in Japan. The meeting consisted of one presidential lecture, two special lectures, two educational lectures, three special symposia, fourteen symposia, three workshops, one battle discussion, and eight luncheon seminars.

More than 130 papers from members were all presented in the poster session where all participants actively joined the presentation and discussion in a small group.

In the special lecture, Prof. Imai from Washington University presented 'NAD world as a novel regulator of ageing and metabolism: a novel measure for the productive aging. In another special lecture, I invited Mr. Kouzou Nishino, the founder of Nishino Method of Breathing, who presented 'The practical method and evidence for Nishino method of breathing'. In the lecture, he presented the cases who dramatically improved osteoporosis with the intervention of Nishino Method.

In the symposia, another important topic of the conference was the metabolic syndrome. The novel screen- ing system for metabolic syndrome was introduced this April in Japan, but the practical methods and follow up system have not yet been established. The presenters actively discussed the methods, the objectives, and various problems that they are facing in the fields, which provided valuable information for the participants.

For the foreign participants, comparative studies and current topics of Anti-Aging in Japan and Europe were specifically presented in the International Session at the first day where three guests from Europe and US and three Japanese members presented in English.

Basic Anti-aging research field also developed recently. Molecular biological analyses with hormones, cytokines, and various biomarkers were presented in the basic science session. However, these data have not yet reduced to the clinical medicine and some time would be needed for the result of basic research to be applied to the clinical medicine.

Authorization as specialists and special institutions led by the Japanese Society of Anti-Aging Medicine have recently helped to broaden the scope of the Anti-Aging association. In Japan, members and specialists are rapidly increasing so that our method and objectives should be globalizing in order to be shared by foreign people in the future.

Takuji Shirasawa, MD Department of Aging Control Juntendo University, Graduate School of Medicine 